\title{
Diagnosis and management of autism in childhood
}

In this clinical review by Stephanie Blenner and colleagues (BMJ 2011;343:d6238, doi:10.1136/bmj.d6238) the authorship and title of reference 4 were wrong. The correct reference is: Ronald A, Hoekstra RA. Autism spectrum disorders and autistic traits: a decade of new twin studies. Am J Med Genet 2011;156B:255-74.

Cite this as: BMJ 2011;343:d7241

๑ BMJ Publishing Group Ltd 2011 